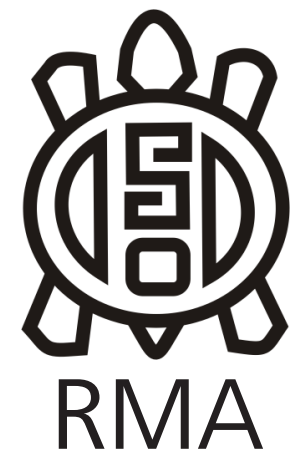

Dossier

\section{Aportes del sitio Hangar al estudio de la gestión tecnológica en la cuenca del Arroyo Salado (provincia de Buenos Aires) durante el Holoceno tardío}

\author{
Contributions of Hangar site to the technological management \\ study in the basin of the Salado creek (province of Buenos Aires) \\ during the late Holocene
}

María Paula Barros*, Jonathan Bellinzoni**, María José Colantonio***,
María Clara Álvarez ${ }^{* * *}$ y Cristian Kaufmann ${ }^{* * * * *}$

*Instituto de Investigaciones Arqueológicas y Paleontológicas del Cuaternario Pampeano (INCUAPA-CONICET). Facultad de Ciencias Sociales, Universidad Nacional del Centro de la Provincia de Buenos Aires. Argentina. E-mail: mapaubarros@gmail.com **Departamento de Arqueología, Facultad de Ciencias Sociales, Universidad Nacional del Centro de la Provincia de Buenos Aires. E-mail: jonabellinzoni@gmail.com ***Departamento de Arqueología, Facultad de Ciencias Sociales, Universidad Nacional delCentro de la Provincia de Buenos Aires. Argentina. E-mail: tresajose2002@yahoo.com.ar ****Instituto de Investigaciones Arqueológicas y Paleontológicas del Cuaternario Pampeano (INCUAPA-CONICET). Facultad de Ciencias Sociales, Universidad Nacional del Centro de la Provincia de Buenos Aires. Argentina. E-mail: malvarez@soc.unicen.edu.ar *****Instituto de Investigaciones Arqueológicas y Paleontológicas del Cuaternario Pampeano (INCUAPA-CONICET). Facultad de Ciencias Sociales, Universidad Nacional del Centro de la Provincia de Buenos Aires. Argentina. E-mail: ckaufman@soc.unicen.edu.ar

\begin{abstract}
Resumen
El sitio Hangar se encuentra emplazado en el partido de General Lamadrid, en el sector noroeste del área Interserrana bonaerense. El conjunto arqueológico se compone de materiales líticos, faunísticos y cerámicos recuperados tanto en posición superficial como en estratigrafía. En este trabajo se presentan los resultados del análisis lítico de los artefactos recolectados en superficie que fueron confeccionados sobre las materias primas más representadas: ftanita, ortocuarcita Grupo Sierras Bayas y chert silíceo. Se reconocieron los objetivos de producción sobre cada una de ellas y se establecieron las etapas de las cadenas operativas. Los resultados muestran un alto porcentaje y variedad de instrumentos, entre los cuales se destacan los raspadores y las puntas de proyectil. En relación con el aprovisionamiento de las rocas, se observan artefactos confeccionados sobre materias primas de la subregión Pampa Húmeda y Pampa Seca. Esto indica la circulación, durante el Holoceno tardío final, de materias primas distantes hasta $400 \mathrm{~km}$ del sitio.
\end{abstract}

Palabras clave: Cazadores-recolectores; Tecnología lítica; Cadena operativa; Movilidad; Gestión de materias primas.

\begin{abstract}
The Hangar site is located in the General Lamadrid county, in the northwest sector of the Interserrana area province of Buenos Aires. The archaeological assemblage is integrated by lithic, faunal, and ceramic materials recovered both in superficial and in stratigraphic position. This paper presents the results of the lithic artifacts collected in the surface, focusing on those that were made on the most represented raw materials, chert, orthoquartzite Sierras Bayas Group, and silicified limestone. The production objectives and the stages of the operational sequence were identified for each raw material. The results show a high percentage and variety of tools, with an important proportion of scrapers and projectile points. In relation to the provisioning of the rocks, artifacts were made of raw materials from the Humid and Dry Pampas sub-regions. This indicates that during the final late Holocene there was a significant circulation of raw materials, distant up to $400 \mathrm{~km}$ away from the site.
\end{abstract}

Keywords: Hunter-gatherers; Lithic technology; Operational sequence; Mobility; Lithic raw material management. 
El sitio Hangar se ubica sobre una pequeña lomada a 170 metros del arroyo Salado, en el partido de General Lamadrid (provincia de Buenos Aires), en el noroeste del área Interserrana (Figura 1). Hasta el momento, las investigaciones arqueológicas indican la presencia de grupos cazadores-recolectores a lo largo del Holoceno medio y tardío. Se ha registrado un total de 19 sitios arqueológicos, tanto superficiales como estratigráficos (Bellinzoni 2018). En el sitio se han realizado tareas de recolección superficial y de excavación. Los restos recuperados incluyen gran cantidad de especímenes líticos, cerámicos y, en menor medida, restos óseos. A partir de los datos cronológicos obtenidos, las ocupaciones fueron asignadas al Holoceno tardío final.

Dentro del conjunto artefactual lítico se ha recuperado una alta proporción de puntas de proyectil y raspadores, entre otras categorías artefactuales. En este contexto, a partir del análisis de las puntas de proyectil asignadas al Holoceno tardío, se ha profundizado acerca de la gestión de las materias primas líticas y de los sistemas de armas utilizados en el sitio (Barros et al. 2018; Kaufmann et al. 2018). Teniendo en cuenta estos resultados, el objetivo de este trabajo es analizar los materiales líticos recuperados en superficie para establecer las cadenas operativas de las materias primas más representadas, la ftanita, la ortocuarcita Grupo Sierras Bayas (GSB) y el chert silíceo.

\section{Aspectos metodológicos}

Los análisis de los materiales líticos se realizarona partir de una aproximación tecno-tipológica (Aschero 1973,

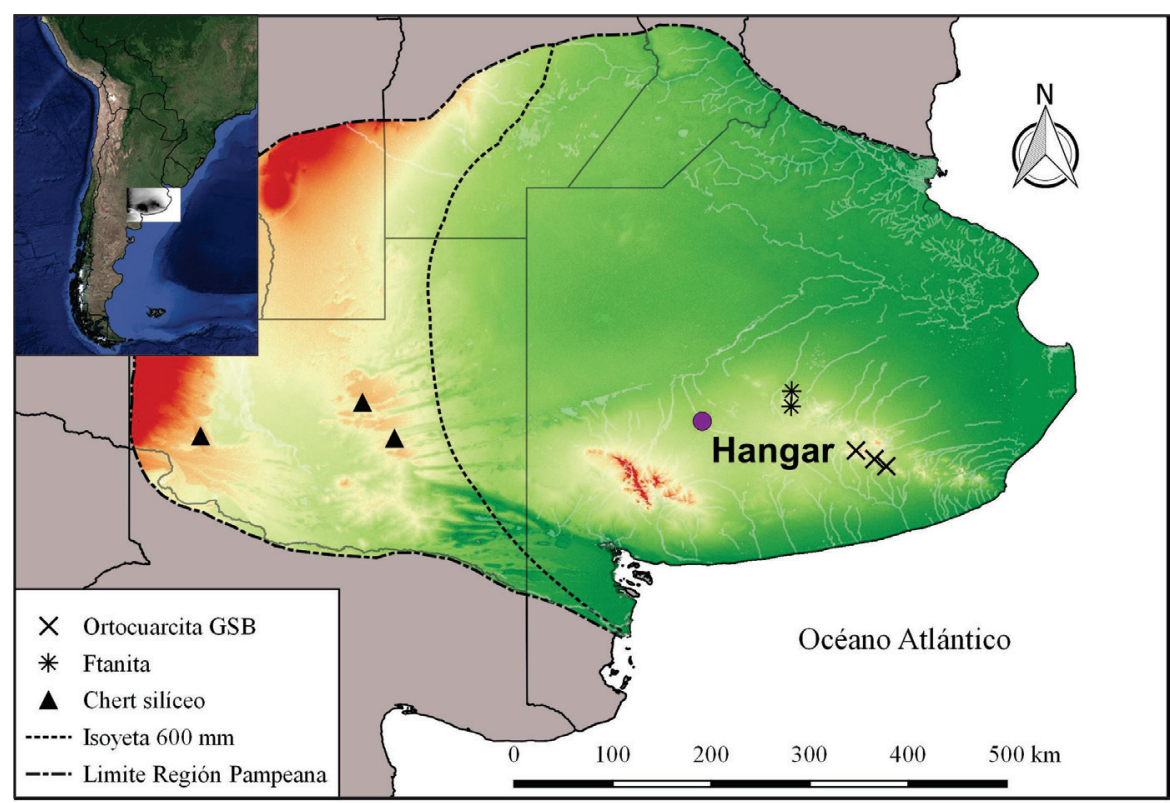

Figura 1. Localización del área de estudio, sitio Hangar y los afloramientos de ftanita, chert silíceo y ortoacuarcita GSB. Referencia: ortocuarcita GSB: Grupo Sierras Bayas.

Figure 1. Study area location, the Hangar site, and outcrops of chert, silicified limestone, and orthoquarzite GSB. Reference: orthoquarzite GSB: Sierras Bayas Group.
1983; Bellelli et al. 1985-1986; Inizan et al. 1995). Además, se reconstruyeron las cadenas operativas de las rocas mayoritarias, siguiendo la propuesta de investigadores de distintas corrientes de la escuela francesa (Boëda 2000; Pelegrin 1995; entre otros). Dentro de este marco, se profundizó en el análisis de los modos de aprovisionamiento, así como en los objetivos de producción, los métodos y técnicas utilizadas durante la talla.

La determinación de las rocas se realizó de forma macroscópica y microscópica, mediante la comparación del material con muestras de mano y cortes delgados que se encuentran en la litoteca del INCUAPA-CONICET. Como resultado, se reconoció la presencia de ftanita, cuyos afloramientos han sido registrados en el sector noroccidental del cordón serrano de Tandilia (Barros y Messineo 2004; Messineo et al. 2004). En segundo lugar, se identificó la ortocuarcita GSB, que se ubica en la porción centro-sur del mismo cordón serrano (Flegenheimer et al. 1996; Colombo 2011; entre otros). Por último, la tercera roca más representada es el chert silíceo, cuyos afloramientos han sido identificados en algunos sectores de la subregión Pampa Seca como Valle Daza (Charlín 2002), Laguna El Carancho y Meseta del Fresco (Berón 2006; Carrera Aizpitarte et al. 2015 Figura I).

\section{Sitio Hangar}

El sitio Hangar (partido de General Lamadrid) fue descubierto durante tareas de prospección llevadas a cabo en el año 2017 en la cuenca media del arroyo Salado. Inicialmente fue identificado por la presencia de materiales en superficie, que fueron expuestos por la acción conjunta de animales de hábitos fosoriales y actividades agropecuarias. En mayo de 2017 se realizaron recolecciones superficiales a lo largo de 11.750 $\mathrm{m}^{2}$, los cuales correspondían a un área sin vegetación y con buena visibilidad. Se efectuaron transectas, a partir de las cuales se recuperaron artefactos líticos, tiestos cerámicos, restos faunísticos y especímenes humanos aislados. Posteriormente, se realizaron ocho sondeos y una excavación de $11 \mathrm{~m}^{2}$ en la cual se hallaron restos arqueológicos, que no son incluidos en este trabajo.

Se realizaron dos fechados 


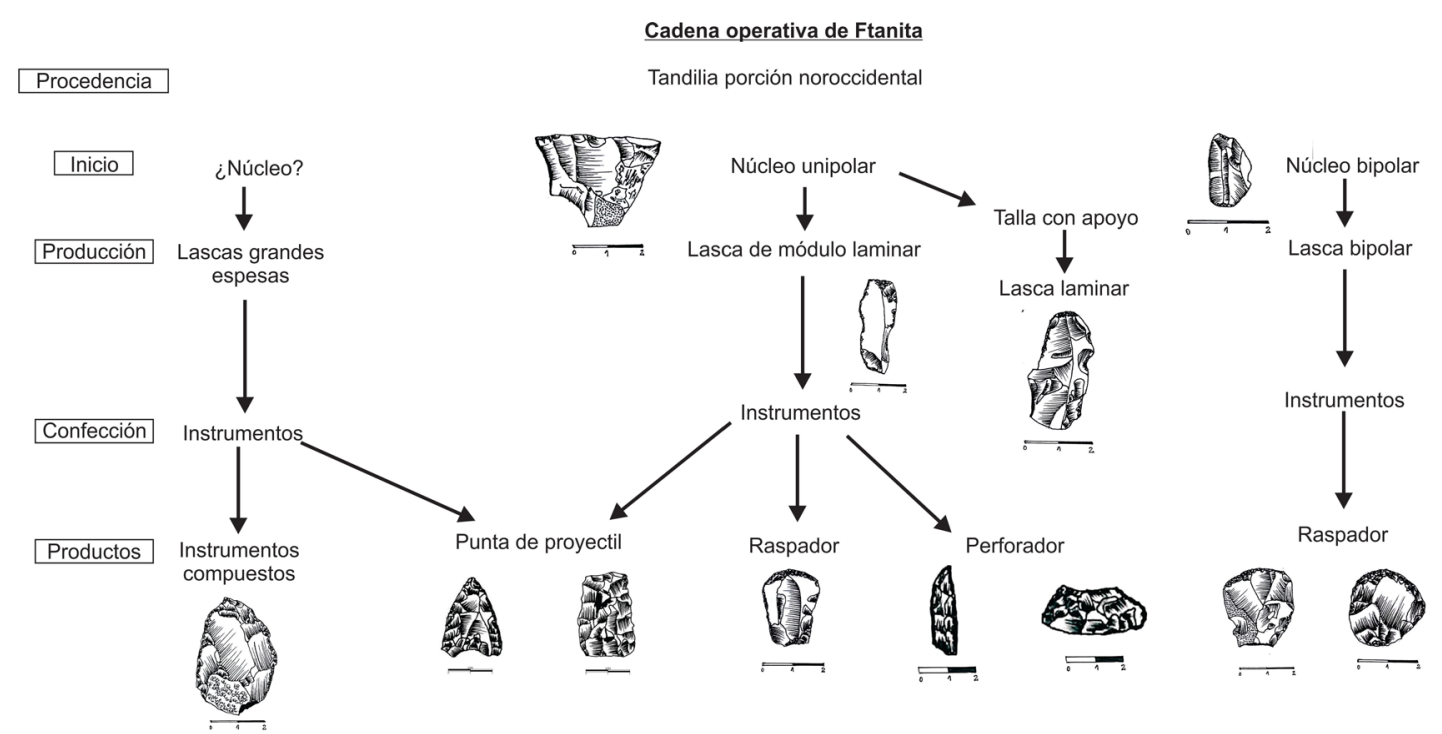

Figura 2. Cadena operativa de ftanita.

Figure 2. Chaîne opératoire of chert

Tabla 1: Categorías artefactuales representadas sobre ftanita, ortocuarcita GSB y chert silíceo.

Table 1. Represented artifactual types on chert, orthoquarzite GSB and silicified limestone.

\begin{tabular}{|c|c|c|c|c|c|c|c|c|c|c|}
\hline \multirow{3}{*}{$\begin{array}{l}\text { Procedencia } \\
\text { materias primas }\end{array}$} & \multirow{3}{*}{ Materia prima } & \multicolumn{7}{|c|}{ Artefactos } & \multirow[b]{3}{*}{ Total } & \multirow[b]{3}{*}{$\%$} \\
\hline & & \multirow{2}{*}{$\begin{array}{c}\text { Instrumentos } \\
\text { tallados }\end{array}$} & \multirow[b]{2}{*}{ Núcleos } & \multicolumn{4}{|c|}{ Productos de talla } & \multirow[b]{2}{*}{ Percutores } & & \\
\hline & & & & LENT & LFCT & LFST & $\begin{array}{l}\text { P.T. } \\
\text { INDI }\end{array}$ & & & \\
\hline $\begin{array}{l}\text { Tandilia-porción } \\
\text { noroccidental }\end{array}$ & Ftanita & 102 & 9 & 189 & 189 & 267 & 1 & 1 & 758 & 58,04 \\
\hline $\begin{array}{l}\text { Tandilia-porción } \\
\text { central }\end{array}$ & $\begin{array}{l}\text { Ortocuarcita } \\
\text { GSB }\end{array}$ & 40 & 0 & 65 & 109 & 179 & 7 & 0 & 400 & 30,62 \\
\hline \multirow[t]{3}{*}{$\begin{array}{l}\text { Sub-región } \\
\text { Pampa Seca }\end{array}$} & Chert silíceo & 16 & 0 & 34 & 41 & 46 & 11 & 0 & 148 & 11,33 \\
\hline & Total & 158 & 9 & 288 & 339 & 492 & 19 & 1 & 1306 & 100 \\
\hline & $\%$ & 12,09 & 0,69 & 22,05 & 25,96 & 37,67 & 1,45 & 0,08 & 100 & \\
\hline
\end{tabular}

Tabla 2. Instrumentos recuperados. Referencias: LRS: Lasca con retoque sumario; LCR: Lasca con rastros complementarios.

Table 2. Recovered instruments. References: LRS: flake with summary edge-retouch; LCR: utilized flake.

\begin{tabular}{|l|c|c|c|c|c|}
\hline \multicolumn{1}{|c|}{ Instrumento } & Ftanita & OGSB & Chert Silíceo & Total & $\%$ \\
\hline Raspadores & 41 & 9 & 6 & 56 & 35,44 \\
\hline Puntas de proyectil & 19 & 8 & 4 & 31 & 19,62 \\
\hline LRS & 21 & 0 & 0 & 21 & 13,29 \\
\hline $\begin{array}{l}\text { Artefacto } \\
\text { compuesto }\end{array}$ & 6 & 7 & 1 & 14 & 8,86 \\
\hline LRC & 2 & 2 & 0 & 4 & 2,53 \\
\hline Fragmento de filo & 6 & 1 & 2 & 9 & 5,70 \\
\hline Raedera & 0 & 6 & 0 & 6 & 3,80 \\
\hline $\begin{array}{l}\text { Perforadores tipo } \\
\text { muleta }\end{array}$ & 2 & 0 & 1 & 3 & 1,90 \\
\hline Pièces esquillées & 3 & 0 & 0 & 3 & 1,90 \\
\hline Biface & 1 & 0 & 1 & 2 & 1.26 \\
\hline Muesca & 1 & 0 & 0 & 1 & 0,63 \\
\hline Cuchillo & 0 & 2 & 1 & 3 & 1,90 \\
\hline Punta destacada & 0 & 1 & 0 & 1 & 0,63 \\
\hline Indeterminado & 0 & 4 & 0 & 4 & 2,53 \\
\hline \begin{tabular}{l} 
Total \\
\hline
\end{tabular} & 102 & 40 & 16 & 158 & 100 \\
\hline
\end{tabular}


radiocarbónicos sobre restos de guanaco con evidencias de procesamiento antrópico, procedentes de la excavación, que arrojaron fechas de $706 \pm 34$ cal años AP (GifA18041 y SacA5357) y 1226 \pm 54 cal años AP (GifA18042 y SacA5357). Estas edades son concordantes con el tipo de registro arqueológico recuperado (e.g., cerámica), que en la región pampeana se vincula sólo con ocupaciones del Holoceno tardío.

\section{Resultados}

En el conjunto lítico de superficie, la roca más representada es la ftanita $(\mathrm{N}=758 ; 52,4 \%)$, seguida por la ortocuarcita GSB ( $\mathrm{N}=400 ; 27,7 \%)$ y el chert silíceo ( $\mathrm{N}=148 ; 10,8 \%)$. Por otra parte, con menor frecuencia se encuentran la metacuarcita, sílice, dolomía silicificada, riolita, cuarzo y calcedonia translúcida, en tanto que el 2,02 \% restante no

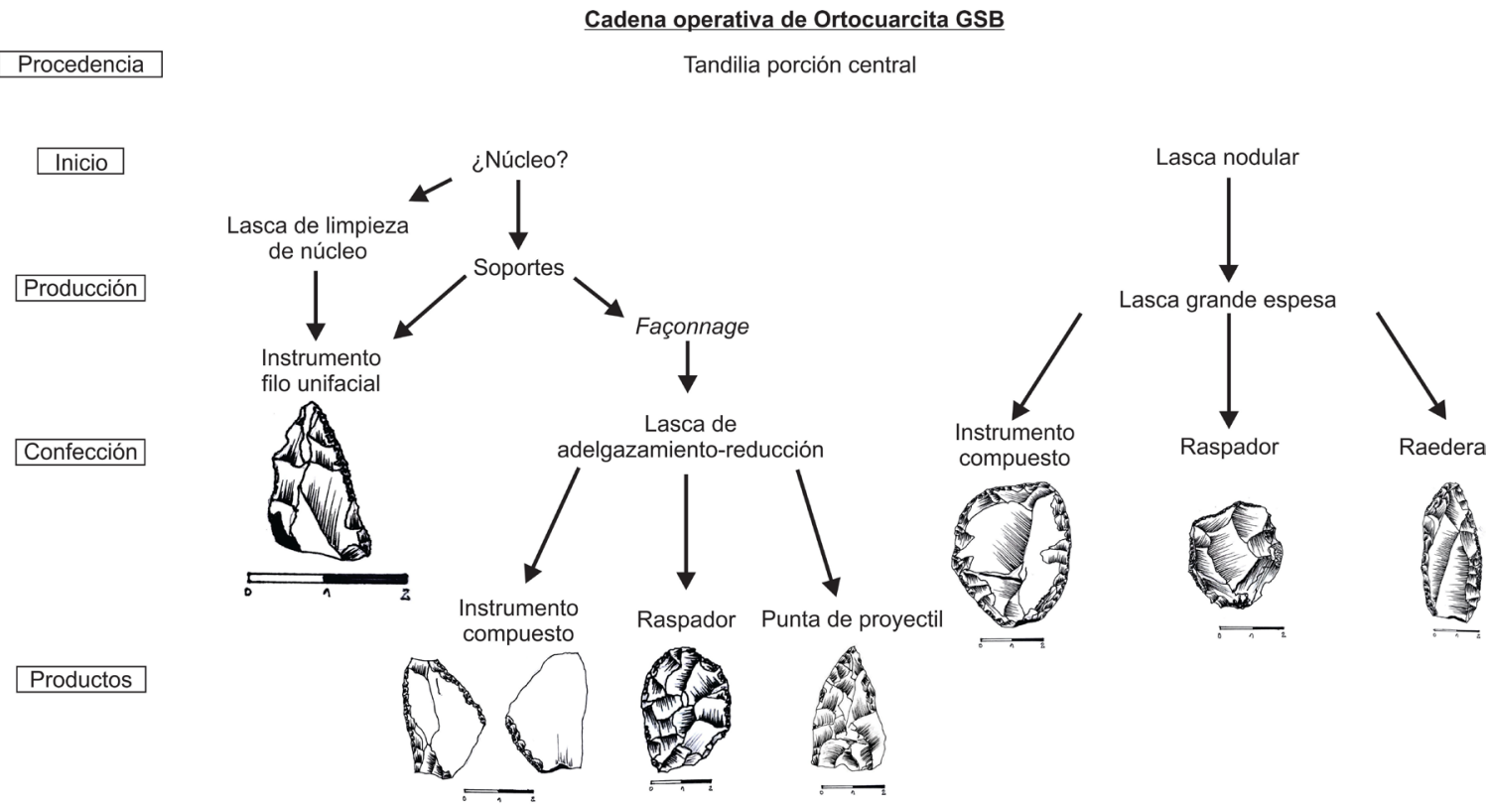

Figura 3. Cadena operativa de ortocuarcita GSB.

Figure 3. Chaîne opératoire of orthoquarzite GSB.

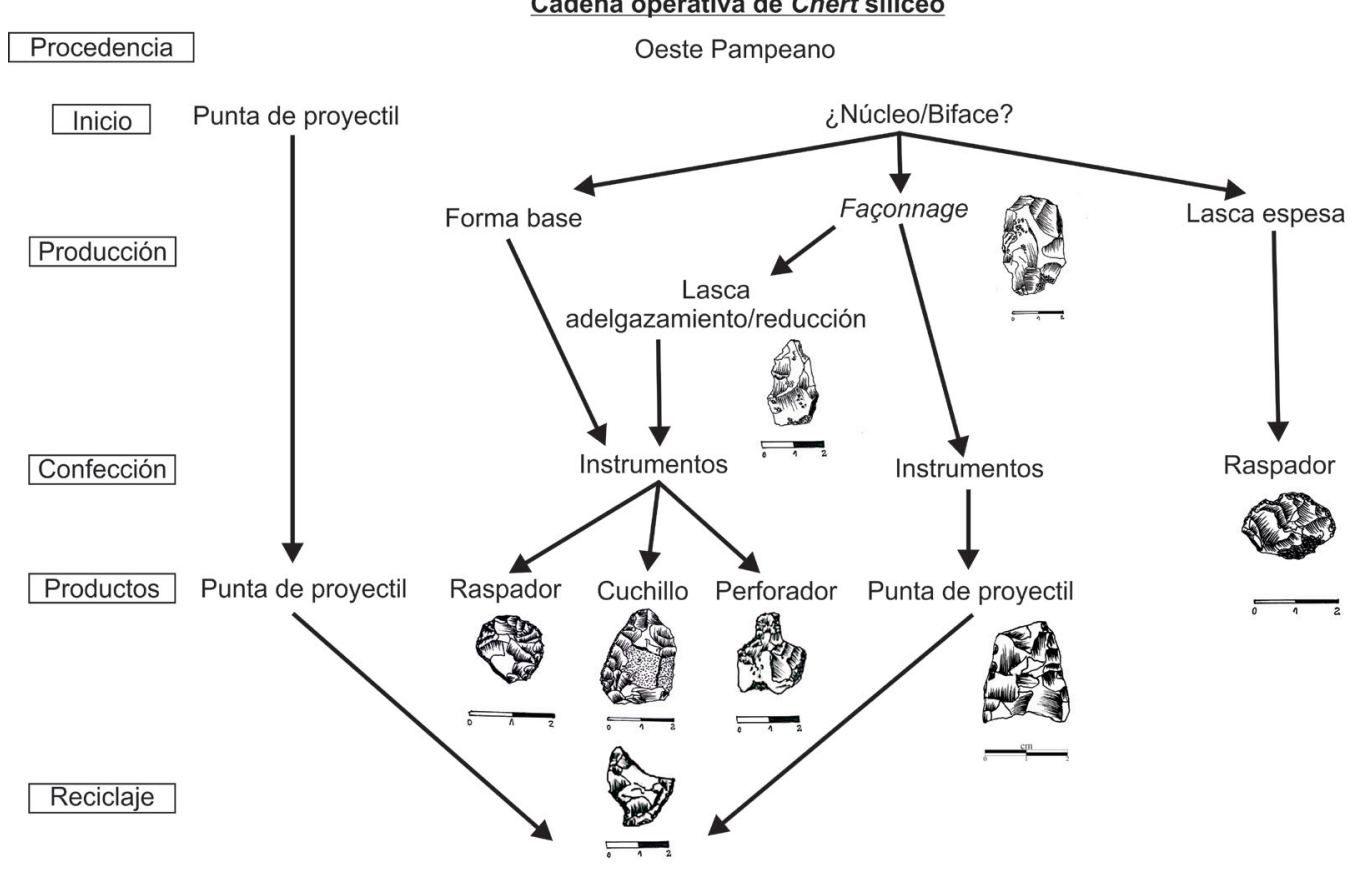

Figura 4. Cadena operativa de chert silíceo.

Figure 4. Chaîne opératoire of silicified limestone. 
pudo ser determinado (ver Tabla 2 en Barros et al. 2018).

Las categorías artefactuales representadas sobre las materias primas mayoritarias son instrumentos, núcleos y productos de talla (Tabla 1). Sobre estas rocas, los objetivos de producción son similares, vinculados principalmente con la fabricación de raspadores y puntas de proyectil. Además, se destaca un importante uso de las lascas de ftanita, que fueron modificadas por retoque sumario (Tabla 2). Por otro lado, se observa la selección de rocas específicas para la fabricación de grupos tipológicos particulares. Este es el caso de las raederas, que fueron confeccionadas sobre ortocuarcita GSB y de los perforadores (tipo muleta) y pièces esquillées, para los cuales se emplearon ftanita y chert silíceo.

\section{Cadena operativa de ftanita}

Esta roca ingresó al sitio bajo la forma de nódulos naturales evidenciado por la presencia de lascas con corteza $(\mathrm{N}=13 ; 2,01 \%$, calculado sobre el total de las lascas) y núcleos unipolares. Los métodos de débitage observados fueron la talla directa (unipolar y centrípeta), con apoyo y bipolar. Los soportes obtenidos fueron seleccionados principalmente para la confección de raspadores y puntas de proyectil. Se observaron distintas formas de producción y manufactura de los raspadores. Esto se evidencia por la selección de distintos tipos de espesores, obtenidos a partir de los diversos métodos de débitage mencionados anteriormente. Otras categorías de instrumentos son perforadores (tipo muleta), pièces esquillées, muescas y un biface. Consideramos que gran parte de los instrumentos fueron confeccionados in situ, dado que presentan cadenas operativas completas (Tabla 2). Además de la obtención de lascas a partir del débitage, se evidencian métodos de façonnage. Estos se observan en la fabricación de puntas de proyectil y en la presencia de lascas de reducción ( $\mathrm{N}=14 ; 2,17 \%)$, adelgazamiento ( $\mathrm{N}=33 ; 5,11 \%)$ y mantenimiento y reactivación de filos $(\mathrm{N}=32 ; 4,96 \%)$.

\section{Cadena operativa de ortocuarcita GSB}

La presencia de una lasca de flanco de núcleo indica que la ortocuarcita GSB podría haber ingresado al sitio como núcleos o como lascas nodulares. Posteriormente, las tareas de talla habrían involucrado la reducción, el adelgazamiento y la formatización de la roca. Se observa que se emplearon métodos de débitage, como la talla directa unipolar y se evidencia gran porcentaje de talla tangencial y bipolar para la obtención de lascas grandes y espesas. Al igual que en el resto de las materias primas, los métodos de façonnage se emplearon para la manufactura de raspadores y puntas de proyectil. Sin embargo, en esta materia prima también se destaca la producción de instrumentos compuestos y raederas, para lo cual se seleccionaron soportes laminares de distintos espesores.

\section{Cadena operativa de chert silíceo}

No se recuperaron núcleos y/o nódulos de chert silíceo en la muestra. A juzgar por la presencia de grandes lascas de adelgazamiento, esta roca podría haber ingresado al sitio en forma de bifaces, además de instrumentos ya formatizados, como puntas de proyectil. Asimismo, a través de la talla unipolar se buscó la producción de soportes, sobre los cuales se confeccionaron diversos grupos tipológicos. Sin embargo, las evidencias indican que en el sitio se habría llevado a cabo principalmente el proceso de façonnage para la manufactura de puntas de proyectil, raspadores y perforadores (tipo muleta).

\section{Discusión y conclusiones}

Los resultados del análisis de los artefactos líticos indican que los grupos humanos que ocuparon el sitio Hangar siguieron objetivos de producción similares en el tratamiento de las tres rocas mayoritarias. Este aspecto es significativo, particularmente si se considera que las materias primas analizadas provienen de diferentes sectores del paisaje y presentan cualidades específicas para la talla. En este sentido, en Hangar se destaca el uso de ftanita (ca. 52,4\%), a diferencia de lo que ocurre en otros conjuntos líticos de la cuenca del arroyo Salado, donde predomina la ortocuarcita GSB a lo largo del Holoceno (Barros et al. 2018). En áreas cercanas, como la cuenca del arroyo Tapalqué, también se registra la explotación de ftanita en mayores frecuencias hacia el Holoceno tardío (ver discusión en Barros y Messineo 2004).

La reconstrucción de las cadenas operativas sugiere que los grupos humanos realizaron partidas logísticas hacia la porción noroccidental y central del sistema serrano de Tandilia para aprovisionarse de ftanita y ortocuarcita GSB. Estas rocas presentan una trayectoria y tratamiento similar, dado que para ambas se emplearon técnicas de talla principalmente directa unipolar y bipolar. No obstante, existen ciertas diferencias en los grupos tipológicos representados, que podrían relacionarse con los tipos de soportes que pueden obtenerse de la ftanita y la mayor versatilidad de la ortocuarcita GSB. Por otro lado, la reconstrucción de la cadena operativa del chert silíceo indica que esta roca habría ingresado al sitio en forma de productos finalizados o bifaces. Las distancias a los afloramientos de esta roca, así como la presencia de perforadores tipo muleta, permiten proponer la obtención del chert silíceo de manera indirecta. Esto indica la presencia de redes de intercambio hacia el Holoceno tardío final, en un contexto social en el cual hubo un aumento de la circulación de bienes, personas e ideas de distintos sectores de la región pampeana (Berón 2006; Mazzanti 2006).

Para concluir, se propone que en el sitio Hangar confluyen los objetivos de producción en las tres materias primas 
analizadas. Este sitio, por su ubicación en el paisaje, se encuentra entre las redes de circulación de los grupos del oeste y el este de la región pampeana, por lo cual presenta importantes frecuencias de materias primas de ambos sectores. Futuros estudios de otros contextos similares del área permitirán poner a prueba las propuestas presentadas en este trabajo.

Olavarría, 8 de noviembre 2019.

\section{Agradecimientos}

Deseamos agradecer a Rocío Torino por la colaboración en el siglado de las piezas. Las investigaciones fueron financiadas por el PICT 2015-0235 dirigido por la Dra. María Clara Álvarez y el PICT 2017-1969 dirigido por el Dr. Cristian Kaufmann. A la Mission Archéologique Française en Argentina, dirigida por Rodolphe Hoguin, por el pago de las dataciones que fueron efectuadas en el Laboratoire des sciences du climat et de l'environnement, Francia. A la Municipalidad de General Lamadrid y a la familia Juarros, por su colaboración en la logística de los trabajos de campo. Este trabajo es parte de las investigaciones del INCUAPA-CONICET (Facultad de Ciencias Sociales, UNICEN). Todo lo expresado y los errores que puedan formar parte de este artículo son de nuestra exclusiva responsabilidad.

\section{Bibliografía}

Aschero, C. A. (1975).Ensayo para una clasificación morfológica de artefactos líticos aplicada a estudios tipológicos comparativos. Informe al CONICET. Facultad de Filosofía y Letras, Universidad de Buenos Aires, Buenos Aires. Manuscrito inédito.

(1983) Ensayo para una clasificación morfológica de artefactos líticos aplicada a estudios tipológicos comparativos. Revisión (Apéndices A y B). Informe al CONICET. Facultad de Filosofía y Letras. Universidad de Buenos Aires, Buenos Aires.Manuscrito inédito.

Barros, M. P. \& P. G. Messineo. (2004). Identificación y aprovisionamiento de chert o ftanita en la cuenca superior del Arroyo Tapalqué. Estudios Atacameños 28: 87-103.

Barros, M. P., Heider, G., Álvarez M. C., Kaufmann, C. \& J. Bellinzoni. (2018). First results of the hunter-gatherer weapon system studies in the middle basin of the Salado creek (Pampas Region, Argentina). Journal of Lithic Studies 5: 1-23. ISSN: 20550472. DOI: http://doi.org/10.2218/jls.2785.

Bellelli, C., A. Guráieb, A. \& J. García. (1985-1987). Propuesta para el análisis y procesamiento por computadora de desechos de talla lítica (DELCO-Desechos líticos computarizados). Arqueología Contemporánea 2 (1): 36-53.

Bellinzoni, J. (2018). Análisis zooarqueológico y tafonómico del sitio Laguna Muscar 2 (Pdo. De General Lamadrid, Pcia. de
Buenos Aires) (pp. 299) (Tesis de licenciatura Inédita). Facultad de Ciencias Sociales. Universidad Nacional del Centro de la Provincia de Buenos Aires.

Berón, M. A. (2006). Base regional de recursos minerales en el occidente pampeano. Procedencia y estrategias de aprovisionamiento. Relaciones de la Sociedad Argentina de Antropología XXXI: 47-88.

Boëda, E. (2000). Les techniques des hommes de la préhistoire pour interroger le présent. Cours d'Eric Boëda: 1-32. Septième École d'été de l'ARCo.

Carrera Aizpitarte, M., M. Berón \& A. Blasi. (2015). Study of siliceous outcrops of Meseta del Fresco, La Pampa, Argentina. Quaternary International 375: 27-43.

Charlin, J. (2002). Aprovisionamiento de Materias Primas Líticas en el N.O. de la Provincia de la Pampa a Fines del Siglo XIX. En: Mazzanti, D., Berón, M. y F. Oliva (Ed), Del Mar a los Salitrales. 10.000 de Historia Pampeana en el Umbral del Tercer Milenio (pp. 205-218).Universidad Nacional de Mar del Plata, Facultad de Humanidades, Laboratorio de Arqueología, Mar del Plata.

Colombo, M. (2011). El área de abastecimiento de las ortocuarcitas del grupo Sierras Bayas y las posibles técnicas para su obtención entre los cazadores y recolectores pampeanos. Intersecciones en Antropología 12: 155-166.

Flegenheimer, N., S. Kain, M. Zárate \& A. Barna.(1996). Aprovisionamiento de cuarcitas en Tandilia, las canteras del Arroyo Diamante. Arqueología 6: 117-141.

Kaufmann, C., Álvarez M. C., Messineo P.G., Barros, M. P., Heider, Guillermo \&M. Bonomo. (2018). Estrategias de caza de guanacos en el sudeste de la región pampeana durante el Holoceno tardío. En Juan Bautista Belardi (Comp). I Taller "Estrategias y tácticas de procuramiento de presas en el pasado: su discusión a partir de la integración de distintas líneas de evidencia" (pp. 41-43). Los Reyunos, Mendoza.

Inizan, M-L., M. Reduron-Ballinger, H. Roche \&J. Tixier. (1995). Technologie de la Pierre taillée (pp.201). CREP con el apoyo de CNRS y Universidad de Paris X Nanterre, Meudon.

Mazzanti, D. (2006). La constitución de territorios sociales durante el Holoceno tardío. El caso de las sierras de Tandilia, Argentina. Relaciones de la Sociedad Argentina de Antropología XXXI: 277-300.

Messineo, P. G.; M. P. Barros; D. G. Poiré y L. Gómez Peral. Características litológicas de los niveles de chert o ftanitas en las Sierras Bayas (Pdo. de Olavarría, Pcia. de Buenos Aires). En: G. Martínez, M. Gutiérrez, R. Curtoni, M. Berón y P. Madrid (Ed) Aproximaciones Contemporáneas a la Arqueología Pampeana. Perspectivas Teóricas, Metodológicas, Analíticas y Casos de Estudio (pp.: 307-319). Olavarría.

Pelegrin, J. (1995). Technologie lithique - Le Châtelperronien de Roc-de-Combe (Lot) et de La Côte (Dordogne). París, C.N.R.S Éditions, Cahiers du Quaternaire, 20. 